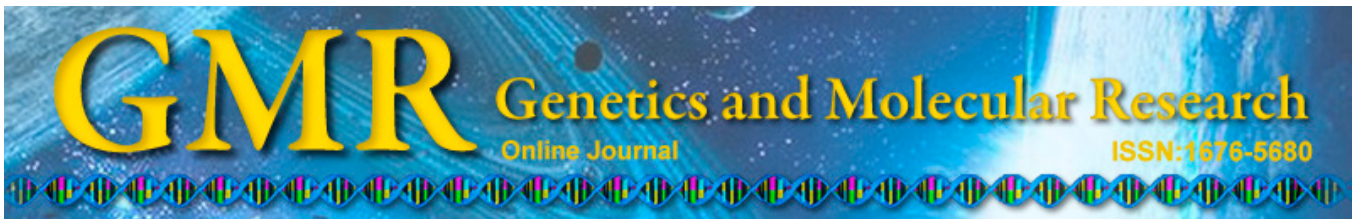

\title{
Associations of miRNA polymorphisms and expression levels with breast cancer risk in the Chinese population
}

\author{
P. Qi ${ }^{1}$, L. Wang ${ }^{2}$, B. Zhou ${ }^{2}$, W.J. Yao ${ }^{3}$, S. Xu ${ }^{1}$, Y. Zhou ${ }^{1}$ and Z.B. Xie ${ }^{1}$ \\ ${ }^{1}$ Department of Head-Neck and Breast Surgery, Xinxiang Central Hospital, \\ Xinxiang, China \\ ${ }^{2}$ Department of General Surgery, \\ The First Affiliated Hospital of Xinxiang Medical University, Xinxiang, China \\ ${ }^{3}$ Department of Thoracic Surgery, \\ The First Affiliated Hospital of Xinxiang Medical University, Xinxiang, China \\ Corresponding author: P. Qi \\ E-mail: 413960747@qq.com
}

Genet. Mol. Res. 14 (2): 6289-6296 (2015)

Received March 3, 2015

Accepted May 14, 2015

Published June 11, 2015

DOI http://dx.doi.org/10.4238/2015.June.11.2

\begin{abstract}
Single-nucleotide polymorphisms in microRNAs (miRNAs) may dramatically affect gene expression and subsequently alter individual susceptibility to cancer, and thus has become a research hotspot for many cancer types, including breast cancer. We recruited 321 breast cancer patients and 290 controls in our study. Four established miRNA single-nucleotide polymorphisms (mir-499 rs3746444 A>G; miR-27a rs895819 A $>$ G; miR-196a2 rs11614913 $\mathrm{T}>\mathrm{C}$; miR-146a rs2910164 G/C) were detected using Taqman assays. Mature miRNA expression, allele distribution, and the association with clinical features were further analyzed. Our results showed that the miR146a rs2910164 G/C polymorphism was associated with an elevated risk of breast cancer (odds ratio $=1.85,95 \%$ confidence interval $=1.03-3.32 ; \mathrm{P}<0.05)$. Compared with the ancestral $\mathrm{T}$ allele
\end{abstract}


in miR-196a2 rs11614913, the variant $\mathrm{C}$ allele was consistently associated with an increased risk of breast cancer (odds ratio $=$ $2.20,95 \%$ confidence interval $=1.19-4.09, \mathrm{P}<0.01)$ and clinical pathological type $(\mathrm{P}<0.01)$. miR-27a rs895819 $\mathrm{A}>\mathrm{G}$ and miR-499 rs3746444 $A>G$ were not associated with breast cancer risk. Analysis of mature miRNA expression confirmed that the variant $\mathrm{C}$ allele in miR146a rs2910164 and miR-196a2 rs11614913 dramatically inhibited production of their mature products. Our results suggested that miR-146a rs2910164 $\mathrm{G}>\mathrm{C}$ and miR-196a2 rs11614913 $\mathrm{T}>\mathrm{C}$ may be biomarkers for predicting breast cancer risk in the Chinese population.

Key words: Breast cancer; MicroRNA; Polymorphism

\section{INTRODUCTION}

Breast cancer is the most frequently diagnosed cancer among women. It is the leading cause of cancer death in less-developed countries (Majeed et al., 2014; Carter, 2014). Because up to $10 \%$ of the total number of women diagnosed with breast cancer report a family history of the disease, microRNAs (miRNAs) may represent a promising new class of potential susceptibility genes (Harquail et al., 2012; Yahya and Elsayed, 2015).

miRNAs are short nucleotide RNAs that influence mRNA stability and translation (Ha and Kim, 2014). Recent studies have demonstrated that miRNAs can act as either tumor suppressors or oncogenes for cancers (Bartel, 2004; Gyparaki et al., 2014) and are associated with numerous other diseases, including heart disease, neurological disorders, and autoimmune conditions. Single-nucleotide polymorphisms (SNPs) are the most common source of genetic polymorphism in the human genome. Various genetic association studies have examined the association between pre-miRNA polymorphisms and cancer risk. SNPs were found to contribute to phenotypic differences in humans (Gutiérrez-Rubio et al., 2015; Mäbert et al., 2014), and sequence variations may affect the processing and target selection of miRNAs in miRNA genes, pri-miRNAs, pre-miRNAs, and mature miRNAs (Kwak et al., 2010).

The most comprehensively studied miRNA polymorphism in breast cancer is miR$196 \mathrm{a} 2$, which is reported to be associated with an increased risk of breast cancer development (Hoffman et al., 2009; O'Day and Lal, 2010). Another study examined the hsa-mir-499 rs3746444 polymorphism in different cancer types and found that it was associated with an increased risk of breast cancer (Wang et al., 2013). Individuals with the variant $G$ allele in the pre-miR-27a rs895819 A/G polymorphism show a reduced risk of breast cancer (Wang et al., 2012). In a previous study, the rs2910164 miR-146a G/C polymorphism showed no association with breast cancer risk (Garcia et al., 2011; Ma et al., 2013).

However, the results of previous studies are controversial and few studies have examined Chinese populations. Thus, we conducted a case control association study to evaluate the association between the pre-miRNA genetic variations mir-499 rs3746444 A>G, miR-27a rs895819 A > G, miR-196a2 rs11614913 T >C, and miR-146a rs2910164 G polymorphisms and the susceptibility to breast cancer. 
miRNA polymorphisms and breast cancer risk

\section{MATERIAL AND METHODS}

\section{Study population}

This study was a population-based case-control study. All samples from study patients were collected during 2011-2013 at the Xinxiang Central Hospital for Breast Cancer Surgery, Henan Province, China. The cases were recruited without any restrictions on age, gender, or disease histology. A total of 321 women with breast cancer were recruited and 290 controls were enrolled as a cancer-free population matched with the cases by age, gender, and residence area.

Each participant participated in a face-to-face interview for a structured questionnaire that elicited information regarding demographic characteristics and health-related factors. After the interview, 3-5 mL venous blood was collected from each participant. The study protocol was approved by the Henan Science Study Association.

\section{SNP selection}

SNPs were selected based on 2 factors. First, the minor allele frequency of selected SNPs was not less than $5 \%$. Second, functional SNPs were selected. To satisfy the 2 criteria, an online miRNA polymorphism database (http://www.patrocles.org/) was searched and the mutation rates and putative target genes were confirmed using 2 additional online databases (http:// www.mirbase.org/ and http://www.ncbi.nlm.nih.gov/snp/). In addition, the related references were searched and the functions of the miRNAs were validated based on previous studies.

\section{Genotype}

Genomic DNA was extracted from white blood cells using the Qiagen Blood Kit (Qiagen, Hilden, Germany) according to the manufacturer protocol. DNA concentration was measured using a Nanodrop ND-1000 system (NanoDrop Technologies, Inc., Wilmington, DE, USA). To analyze rs11614913 in miR-196-a2, rs895819 in hsa-miR-27a, and rs2910164 in miR-146a, real-time polymerase chain reaction (PCR) allelic discrimination was performed with Step-One Real-Time PCR apparatus (Applied Biosystems, Foster City, CA, USA) using standard TaqMan genotyping assays according to the manufacturer instructions. Briefly, probes, primers, and TaqMan universal PCR Master Mix were obtained from Applied Biosystems. A reaction solution of $10 \mu \mathrm{L}$ contained $0.5 \mu \mathrm{L}$ TaqMan Genotyping Assay mix (consisting of 20X unlabeled PCR primers and TaqMan minor groove binder probe, 6-carboxyfluorescein, and a VIC dye-labeled probe), $8 \mu \mathrm{L}$ PCR mixture reagent, and $10 \mathrm{ng}$ genomic DNA. Reactions were conducted according to manufacturer instructions. The PCR consisted of pre-PCR read at $60^{\circ} \mathrm{C}$ for $30 \mathrm{~s}$; holding stage at $95^{\circ} \mathrm{C}$ for $10 \mathrm{~min}$; and 50 cycles of denaturing at $92^{\circ} \mathrm{C}$ for 15 $\mathrm{s}$, annealing $60^{\circ} \mathrm{C}$ for $1 \mathrm{~min} 30 \mathrm{~s}$, and post-PCR read at $60^{\circ} \mathrm{C}$ for $30 \mathrm{~s}$. All primers are displayed in Table 1.

\section{miRNA expression}

Total RNA was extracted using TRIzol Reagent (Invitrogen, Carlsbad, CA, USA) and stored at $-80^{\circ} \mathrm{C}$. The quantitative real-time PCR was performed using a 2-step method accord- 
ing to manufacturer instructions (Takara, Shiga, Japan). Real-time PCR was conducted using the ABI 7900 fast sequence detection system (Applied Biosystems) with the SYBR green fluorescent dye. The cycling parameters were as follows: $95^{\circ} \mathrm{C}$ for $10 \mathrm{~min}$ followed by 40 cycles of $95^{\circ} \mathrm{C}$ for $15 \mathrm{~s}, 60^{\circ} \mathrm{C}$ for $1 \mathrm{~min}$, and elongation at $72^{\circ} \mathrm{C}$ for $30 \mathrm{~s}$. The human $\beta$-actin transcript was used as an internal reference to control for variations in the total mRNA quantity of each sample. Each RNA sample was analyzed in triplicate using the following primers:

\begin{tabular}{ll}
\multicolumn{1}{c}{ Table 1. Primers for miRNA. } & \\
\hline Gene SNP & Primers \\
\hline mir-499 rs3746444 & Forward: CAAAGTCTTCACTTCCCTGCCA \\
miR-27a rs895819 & Reverse: GATGTTTAACTCCTCTCCACGTGATC \\
miR-196a2 rs11614913 & Forward: GAACTTAGCCACTGTGAACACCACTTGG \\
miR-146a rs2910164 & Reverse: TTGCTTCCTGTCACAAATCACATTG \\
& Forward: CCAGGCTGGTCTCGAACTC \\
& Reverse: CTGAATAAATGAGTTCTGCAAAACAGGTT \\
& Forward: CATGGGTTGTGTCAGTGTCAGAGCT \\
\hline
\end{tabular}

Human miR-196a primers: forward 5'-GAGGCGTGGCAGACTATGC-3'; reverse 5'CTTGTACTCCGTCAGCGTGA-3'; human miR-146a primers: forward 5'-TGTAACCAGAG AGCGGGATGT-3'; reverse 5'-TTTTGGCATAACTAAGGCCGAA-3'; human $\beta$-actin primers: forward5'-CACTCTTCCAGCCTTCCTTC-3'; reverse 5'-GGATGTCCACGTCACACTTC-3'.

\section{Statistical analysis}

Hardy-Weinberg equilibrium was tested for each polymorphism using the $\chi^{2}$ test in patients and controls. Allelic frequencies were estimated and differences in allele frequencies between case and control subjects were tested using likelihood ratio $\chi^{2}$ tests. The homozygote of the most frequent allele was used as a reference to calculate the odds ratio (OR). For an OR and $95 \%$ confidence interval $(95 \% \mathrm{CI})$, logistical regression was used based on a model for sex and age of the patients. Data were analyzed using SPSS 13.0 (SPSS, Inc., Chicago, IL, USA). Values of $\mathrm{P}<0.05$ were considered to be statistically significant.

\section{RESULTS}

\section{Clinical characteristics of study population}

In the case group (Table 2), fewer subjects reported with estrogen receptor- and progesterone receptor-negative status. Ductal type was the common pathological type. Most patients were in stage II or III. The age distribution in all study patients was in the same level.

\section{Association between miRNA polymorphisms and breast cancer susceptibility}

Overall, 321 women with breast cancer and 290 controls without breast cancer were recruited in this study. The distribution of miRNA genotypes and their association with breast cancer risk is showed in Table 3. Compared with the TT genotypes, carriers with the pre- 
miR-196 CT and CC genotypes showed a significant association with an increased breast cancer risk $(\mathrm{OR}=1.49,95 \% \mathrm{CI}=1.05-2.10$ and $\mathrm{OR}=2.20,95 \% \mathrm{CI}=1.19-4.09$, respectively $)$. Carriers with the pre-miR-146a CC genotypes also showed a significant association with increased breast cancer risk $(\mathrm{OR}=1.85,95 \% \mathrm{CI}=1.03-3.32)$. No significant association was observed between the other miRNA polymorphisms and breast cancer risk.

\begin{tabular}{lcc} 
Table 2. Clinical parameters of the study population. & & \\
\hline & Case & Percentage (\%) \\
\hline Age & & 53.89 \\
$\leq 50$ & 173 & 46.11 \\
$>50$ & 148 & 61.68 \\
Pathological type & & 38.32 \\
Ductal & 198 & 19.94 \\
Others & 123 & 33.64 \\
TNM stage & & 28.04 \\
I & 64 & 18.38 \\
II & 108 & 63.55 \\
III & 90 & 36.45 \\
IV & 59 & 61.37 \\
Estrogen receptor & & 38.63 \\
Positive & 204 & \\
Negative & 117 & 52.65 \\
Progesterone receptor & & 47.35 \\
Positive & 197 & \\
Negative & 124 & \\
Her2 status & & \\
Positive & 169 & \\
Negative & 152 & \\
\hline
\end{tabular}

Table 3. Association between miRNA polymorphisms and breast cancer susceptibility.

\begin{tabular}{|c|c|c|c|c|}
\hline Genotype & Case & Control & Adjusted OR & $P$ value \\
\hline \multicolumn{5}{|l|}{ miR-499 } \\
\hline AA & 152 & 141 & 1 (Ref) & \\
\hline GA & 117 & 112 & $0.93(0.66-1.32)$ & 0.73 \\
\hline GG & 52 & 37 & $1.26(0.78-2.03)$ & 0.34 \\
\hline \multicolumn{5}{|l|}{ miR-27a } \\
\hline $\mathrm{AA}$ & 101 & 95 & 1 (Ref) & \\
\hline $\mathrm{AG}$ & 159 & 139 & $0.68(0.75-1.54)$ & 0.58 \\
\hline GG & 61 & 56 & $1.02(0.64-1.62)$ & 0.92 \\
\hline \multicolumn{5}{|l|}{ miR-196a } \\
\hline TT & 168 & 185 & 1 (Ref) & \\
\hline СТ & 119 & 88 & $1.49(1.05-2.10)^{*}$ & 0.03 \\
\hline $\mathrm{CC}$ & 34 & 17 & $2.20(1.19-4.09)^{*}$ & 0.01 \\
\hline \multicolumn{5}{|l|}{ miR-146a } \\
\hline GG & 146 & 126 & 1 (Ref) & \\
\hline $\mathrm{GC}$ & 132 & 144 & $0.79(0.56-1.11)$ & 0.17 \\
\hline $\mathrm{CC}$ & 43 & 20 & $1.85(1.03-3.32)^{*}$ & 0.04 \\
\hline
\end{tabular}

\section{Association between miRNA expression level and SNP distribution}

The expression of miR196a and miR146a was analyzed in selected tumor samples using real-time PCR (Figure 1). The miR196a and miR146a level was dramatically decreased $(\mathrm{P}<0.05)$ in the samples with $\mathrm{CC}$ variants compared with the TT variant. 
A

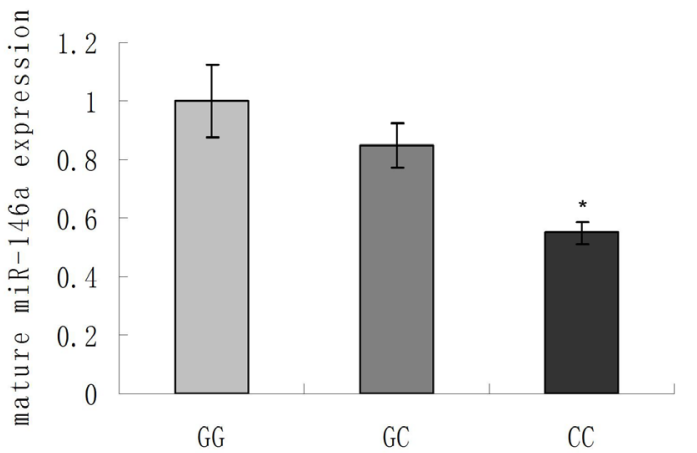

B

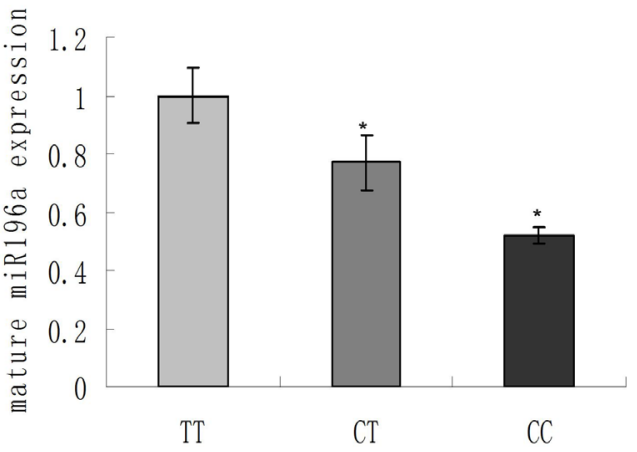

Figure 1. Association between miRNA expression and SNP distribution in the study population. A. miR-146a expression and rs11614913 T>C. B. miR-196a expression and rs2910164. All experiments were tested in triplicate. $\mathrm{P}<0.05$ compared with wild-type genotype.

\section{Association between miRNA SNPs and clinical features}

The clinical features associated with this SNP are shown in Table 4. No significant association was observed among the miR27a, miR146a, and miR499 polymorphisms and clinic pathologic parameters, including estrogen and progesterone receptors, and human growth factor receptor 2. However, an association between miR196a variant and clinical pathological types was observed $(\mathrm{P}<0.01)$.

\begin{tabular}{|c|c|c|c|c|c|c|c|c|c|c|c|c|c|}
\hline \multirow[b]{2}{*}{ No. } & \multirow[t]{2}{*}{ Case } & \multicolumn{2}{|c|}{$\operatorname{miR}-499$} & \multirow[t]{2}{*}{$\mathrm{P}$} & \multicolumn{2}{|c|}{$\operatorname{miR}-27 \mathrm{a}$} & \multirow[t]{2}{*}{$\mathrm{P}$} & \multicolumn{2}{|c|}{ miR-196a } & \multirow[t]{2}{*}{$\mathrm{P}$} & \multicolumn{2}{|c|}{ miR-146a } & \multirow[t]{2}{*}{$\mathrm{P}$} \\
\hline & & 152 & 169 & & 101 & 210 & & 168 & 153 & & 146 & 175 & \\
\hline Age & & $\mathrm{AA}$ & GA/GG & & AA & $\mathrm{AG} / \mathrm{GG}$ & & TT & $\mathrm{CT} / \mathrm{CC}$ & & GG & $\mathrm{GC} / \mathrm{CC}$ & 0.29 \\
\hline$\leq 50$ & 173 & 86 & 87 & 0.35 & 51 & 122 & 0.41 & 89 & 84 & 0.72 & 74 & 99 & \\
\hline$>50$ & 148 & 66 & 82 & & 50 & 98 & & 79 & 69 & & 72 & 76 & \\
\hline \multicolumn{14}{|c|}{ Pathological type } \\
\hline Ductal & 198 & 101 & 97 & 0.09 & 60 & 138 & 0.57 & 72 & 126 & $<0.01$ & 94 & 104 & 0.4 \\
\hline Others & 123 & 51 & 72 & & 41 & 82 & & 96 & 27 & & 52 & 71 & \\
\hline \multicolumn{14}{|c|}{ Estrogen receptor } \\
\hline Positive & 204 & 98 & 106 & 0.74 & 57 & 147 & 0.07 & 103 & 101 & 0.38 & 89 & 115 & 0.37 \\
\hline Negative & 117 & 54 & 63 & & 44 & 73 & & 65 & 52 & & 57 & 60 & \\
\hline \multicolumn{14}{|c|}{ Progesterone receptor } \\
\hline Positive & 197 & 92 & 105 & 0.76 & 55 & 142 & 0.08 & 101 & 96 & 0.63 & 86 & 111 & 0.41 \\
\hline Negative & 124 & 60 & 64 & & 46 & 78 & & 67 & 57 & & 60 & 64 & \\
\hline \multicolumn{14}{|l|}{ Her 2 status } \\
\hline Positive & 169 & 79 & 90 & 0.82 & 56 & 113 & 0.49 & 92 & 77 & 0.42 & 81 & 88 & 0.35 \\
\hline Negative & 152 & 73 & 79 & & 45 & 107 & & 76 & 76 & & 65 & 87 & \\
\hline
\end{tabular}

\section{DISCUSSION}

The present case-control study evaluated the potential association between 4 SNPs (rs2910164, rs11614913, rs3746444, and rs895819) in pre-miRNAs and 321 breast cancer 
patients and 290 controls in a Chinese population. We found that the heterozygous variant of miR-146a G/C was significantly associated with an increased risk of breast cancer. In contrast, no significant difference was observed in the distribution of miR-196a T/C (rs11614913) and miR-499 A>G (rs3746444) polymorphisms in any groups, both at the genotype and the allele levels. Additionally, miRNA expression analysis showed that the SNPs in miR196 and miR146 significantly impaired mature miRNA expression and may have contributed to target gene function.

The miR-146a rs2910164 polymorphism occurs at the first nucleotide of pre-miR$146 \mathrm{a}$, with a $\mathrm{G}$ to $\mathrm{C}$ change in the passenger strand, resulting in a change from the $\mathrm{G}: \mathrm{U}$ pair to a $\mathrm{C}: \mathrm{U}$ mismatch in the stem of the miR-146a precursor and reduced production of mature miR146a (Kupcinskas et al., 2014). Jazdzewski et al. (2008) found that the miR-146a rs2910164 $\mathrm{CC}$ genotype in pre-miR-146a was associated with decreased mature miR-146a expression and increased reporter gene expression, including genes involved in the Toll-like receptor and cytokine signaling pathways.

miR-196a 2 is composed of 2 different mature miRNAs, miR-196a-5P and miR196a-3P, which are processed from the same stem-loop (Chen et al., 2011). rs11614913 is located in the mature sequence of miR-196a-3P and may lead to less efficient processing of the miRNA precursor to its mature form, as well as diminish the capacity to regulate target genes. Hoffman et al. (2009) found that miR-196a2 rs11614913 not only influences the mature miR196a levels, but also has a phenotypic effect on target gene expression, as a total of 684 transcripts were altered expression following introduction of pre-miR-196a-C, and fewer than half of that number were significantly altered after introduction of pre-miR-196a-T (Wang et al., 2012). Numerous studies have shown that individuals carrying the CC genotype show a significantly increased risk of breast cancer, lung cancer, gastric cancer, colorectal cancer, and hepatocellular carcinoma compared to those with the TT or TT+TC genotypes ( $\mathrm{Li}$ et al., 2010; Lee et al., 2014).

An SNP in the pre-miRNA region of hsa-mir-499 (rs3746444: A>G) was detected in a cell line of invasive breast cancer. Hu et al. (2009) reported that the hsa-mir-499 variant genotype was associated with a significantly increased risk of breast cancer; in contrast, Catucci et al. (2010) reported no association among their total of 1894 breast cancer cases who were negative for disease-causing mutations or unclassified variants in BRCA1 and BRCA2, and 2760 controls from Germany and Italy.

The SNP rs895819, located in pre-miR-27a, was evaluated in an association study in which SNPs located in recently identified breast cancer-relevant miRNA genes were examined (including pre-miRNAs and an approximately 200-base pair flanking region) by sequencing of these regions (Slaby et al., 2012). In a group of 1217 German familial breast cancer patients and 1422 unrelated healthy German women, Yang et al. (2010) found that the G allele of rs895819, located in the terminal loop of a pre-miR-27a oncogene, was associated with a reduced familial breast cancer risk. We found no association between these 2 SNPs and breast cancer risk. The inconsistency of these observations may be explained by the different ethnicities of the investigated populations.

In conclusion, our data suggest that a common polymorphism in pre-miR-146a affects miR expression, contributes to the genetic predisposition to breast cancer, and plays a role in the tumorigenesis through somatic mutation. Preliminary evidence suggests that these effects are mediated through target genes whose expression is affected by SNP status. 


\section{Conflicts of interest}

The authors declare no conflict of interest.

\section{ACKNOWLEDGMENTS}

The authors received no financial support for the research, authorship, and/or publication of this article.

\section{REFERENCES}

Bartel DP (2004). MicroRNAs: genomics, biogenesis, mechanism, and function. Cell 116: 281-297.

Carter D (2014). New global survey shows an increasing cancer burden. Am. J. Nurs. 114: 17.

Catucci I, Yang R, Verderio P, Pizzamiglio S, et al. (2010). Evaluation of SNPs in miR-146a, miR196a2 and miR-499 as low-penetrance alleles in German and Italian familial breast cancer cases. Hum. Mutat. 31: E1052-E1057.

Chen C, Zhang Y, Zhang L, Weakley SM, et al. (2011). MicroRNA-196: critical roles and clinical applications in development and cancer. J. Cell Mol. Med. 15: 14-23.

Garcia AI, Cox DG, Barjhoux L, Verny-Pierre C, et al. (2011). The rs2910164 G>C SNP in the miR146a gene is not associated with breast cancer risk in BRCA1 and BRCA2 mutation carriers. Hum. Mutat. 32: 1004-1007.

Gutiérrez-Rubio SA, Quintero-Ramos A, Durán-Cárdenas A, Franco-Topete RA, et al. (2015). 1236 C/T and 3435 C/T polymorphisms of the $A B C B 1$ gene in Mexican breast cancer patients. Genet. Mol. Res. 14: 1250-1259.

Gyparaki MT, Basdra EK and Papavassiliou AG (2014). MicroRNAs as regulatory elements in triple negative breast cancer. Cancer Lett. 354: 1-4.

Ha M and Kim VN (2014). Regulation of microRNA biogenesis. Nat. Rev. Mol. Cell Biol. 15: 509-524.

Harquail J, Benzina S and Robichaud GA (2012). MicroRNAs and breast cancer malignancy: an overview of miRNAregulated cancer processes leading to metastasis. Cancer Biomark. 11: 269-280.

Hoffman AE, Zheng T, Yi C, Leaderer D, et al. (2009). microRNA miR-196a-2 and breast cancer: a genetic and epigenetic association study and functional analysis. Cancer Res. 69: 5970-5977.

Hu Z, Liang J, Wang Z, Tian T, et al. (2009). Common genetic variants in pre-microRNAs were associated with increased risk of breast cancer in Chinese women. Hum. Mutat. 30: 79-84.

Jazdzewski K, Murray EL, Franssila K, Jarzab B, et al. (2008). Common SNP in pre-miR-146a decreases mature miR expression and predisposes to papillary thyroid carcinoma. Proc. Natl. Acad. Sci. U. S. A. 105: 7269-7274.

Kupcinskas J, Bruzaite I, Juzenas S, Gyvyte U, et al. (2014). Lack of association between miR-27a, miR-146a, miR196a-2, miR-492 and miR-608gene polymorphisms and colorectal cancer. Sci. Rep. 4: 5993.

Kwak PB, Iwasaki S and Tomari Y (2010). The microRNA pathway and cancer. Cancer Sci. 101: 2309-2315.

Lee SJ, Seo JW, Chae YS, Kim JG, et al. (2014). Genetic polymorphism of miR-196a as a prognostic biomarker for early breast cancer. Anticancer Res. 34: 2943-2949.

Li XD, Li ZG, Song XX and Liu CF (2010). A variant in microRNA-196a2 is associated with susceptibility to hepatocellular carcinoma in Chinese patients with cirrhosis. Pathology 42: 669-673.

Ma F, Zhang P, Lin D, Yu D, et al. (2013). There is no association between microRNA gene polymorphisms and risk of triple negative breast cancer in a Chinese Han population. PLoS One 8: e60195.

Mäbert K, Cojoc M, Peitzsch C, Kurth I, et al. (2014). Cancer biomarker discovery: current status and future perspectives. Int. J. Radiat. Biol. 90: 659-677.

Majeed W, Aslam B, Javed I, Khaliq T, et al. (2014). Breast cancer: major risk factors and recent developments in treatment. Asian Pac. J. Cancer Prev. 15: 3353-3358.

O’Day E and Lal A (2010). MicroRNAs and their target gene networks in breast cancer. Breast Cancer Res. 12: 201.

Slaby O, Bienertova-Vasku J, Svoboda M and Vyzula R (2012). Genetic polymorphisms a microRNAs: new direction in molecular epidemiology of solid cancer. J. Cell Mol. Med. 16: 8-21.

Wang J, Wang Q, Liu H, Shao N, et al. (2012). The association of miR-146a rs2910164 and miR-196a2 rs11614913 polymorphisms with cancer risk: a meta-analysis of 32 studies. Mutagenesis 27: 779-788.

Wang PY, Gao ZH, Jiang ZH, Li XX, et al. (2013). The associations of single nucleotide polymorphisms in miR-146a, miR-196a and miR-499 with breast cancer susceptibility. PLoS One 8: e70656.

Yahya SM and Elsayed GH (2015). A summary for molecular regulations of miRNAs in breast cancer. Clin. Biochem. 388-396.

Yang R, Schlehe B, Hemminki K, Sutter C, et al. (2010). A genetic variant in the pre-miR-27a oncogene is associated with a reduced familial breast cancer risk. Breast Cancer Res. Treat. 121: 693-702. 